\title{
A multidimensional approach to the management of severe asthma: Inflammometry, molecular microbiology and bronchial thermoplasty
}

\author{
Conor Cox BScH MBBCh ${ }^{1}$, Melanie Kjarsgaard RRT ${ }^{1}$, Michael G Surette PhD², \\ P Gerard Cox MBBCh FRCPI FRCPC ${ }^{1}$, Parameswaran Nair MD PhD FRCP FRCPC ${ }^{1}$
}

\begin{abstract}
C Cox, M Kjarsgaard, MG Surette, PG Cox, P Nair. A multidimensional approach to the management of severe asthma: Inflammometry, molecular microbiology and bronchial thermoplasty. Can Respir J 2015;22(4):221-224.
\end{abstract}

The authors illustrate the merits of identifying the components of diseases (eg, bronchitis and airway hyper-responsiveness) that contribute to exacerbations in the management of a patient with severe asthma. Quantitative cell counts in sputum identified a neutrophilic - as opposed to eosinophilic bronchitis that enabled a stepwise weaning of prednisone. Molecular microbiology and extended culture methods identified anaerobes and other airway microbiome that helped to guide the use of antibiotics. Further control of asthma was achieved by performing bronchial thermoplasty.

Key Words: Molecular microbiology; Severe asthma; Sputum cell counts; Thermoplasty

T he cornerstone of asthma management is inhaled corticosteroids and short-acting bronchodilators. As asthma becomes more severe and poorly controlled, treatment is escalated by increasing the dose of steroids and by adding other controller medications, such as long-acting bronchodilators, and other drugs with anti-inflammatory effects such as leukotriene receptor antagonists, prednisone or monoclonal antibodies directed against immunoglobulin E (1). These decisions are based on assessments of airflow and symptoms. The other components of airway disease (ie, bronchitis and airway hyperresponsiveness [2]) are not often measured to guide therapy. In the present report, we illustrate the merits of this strategy by describing the management of severe asthma in a patient who experienced frequent exacerbations despite being on daily maintenance high doses of prednisone and bronchodilators. Quantitative cell counts in sputum identified a neutrophilic - as opposed to eosinophilic - bronchitis that enabled stepwise weaning of prednisone. Molecular microbiology and extended culture methods identified anaerobes and other airway microbiome that helped to guide the use of antibiotics. Further control of asthma was achieved by performing bronchial thermoplasty.

\section{CASE PRESENTATION}

A 41-year-old man was referred in 2009 by a pulmonary specialist for further evaluation of his severe persistent asthma refractory to treatment. He had a high burden of illness, with clinic appointments every four months, unscheduled hospital and emergency room visits, and at least four exacerbations of bronchitis per year. He was diagnosed with asthma at a young age, which was always very difficult to control. His main symptoms were dyspnea on exertion, dizzy spells, cough and sputum, and frequent asthma exacerbations that required courses of prednisone and high maintenance doses of inhaled corticosteroid. Aggravating factors included stress, heat, poor air quality, strong fragrances and second-hand smoke. There was no history of childhood bronchiolitis and no inciting pathogens had ever been identified,

\section{Une approche multidimensionnelle de la prise en charge de l'asthme sévère : l'inflammométrie, la microbiologie moléculaire et la thermoplastie bronchique}

Les auteurs exposent les avantages de déterminer les aspects de la maladie (p. ex., bronchite et hyperréactivité des voies respiratoires) qui contribuent aux exacerbations pour prendre en charge un patient atteint d'asthme sévère. La numération cellulaire quantitative des expectorations, qui a permis de diagnostiquer une bronchite à neutrophiles plutôt qu'à éosinophiles, a favorisé un sevrage graduel de la prednisone. La microbiologie moléculaire et des modes de culture étendus ont permis de repérer les anaérobies et un autre microbiome des voies aériennes qui ont contribué à orienter l'utilisation des antibiotiques. La thermoplastie bronchique a permis de mieux contrôler l'asthme.

although sputum had appeared purulent on many occasions. He had never been intubated or mechanically ventilated; however, in 1999 , he experienced a pneumomediastinum and pneumothoraces, possibly secondary to severe bronchospasm and airflow obstruction. His only comorbidities were gastroesophageal reflux disease, managed with esomeprazole, and gout. He had a five pack-year smoking history but had quit smoking $>10$ years previously. At the time of assessment, he was on budesonide $200 \mu \mathrm{g}+$ formoterol $6 \mu \mathrm{g}$, eight to 12 puffs daily, tiotropium $18 \mu \mathrm{g}$ daily, theophylline $200 \mathrm{mg}$ daily, N-acetylcysteine $500 \mathrm{mg}$ twice daily, and additional salbutamol metered dose inhaler $100 \mu \mathrm{g}$ two to six puffs daily. He had just completed a seven-day course of prednisone $40 \mathrm{mg}$ daily - his 12 th course in as many months - and was on his usual maintenance dose of $25 \mathrm{mg}$ daily. He had tried almost every medical therapy that has been approved in Canada; however, his asthma remained refractory to treatment. He had never been treated with omalizumab because he was not atopic.

At the time of assessment in the authors' clinic, he was not in distress and was breathing quietly. His body mass index was $38.25 \mathrm{~kg} / \mathrm{m}^{2}$. He had a well-healed nasal fracture with minor deformity but clear nostrils. Examination of the lower respiratory system was normal and there were no signs of chest overinflation. Cardiovascular examination was unremarkable except for a heart rate of 52 beats/min, but there were no murmurs or bruits. There were no findings on abdominal examination, nor did he exhibit any thyroid swelling or neurological abnormalities. Examination of his oropharynx, head and neck were noncontributory. There was no clinical, radiological or laboratory evidence to suggest pulmonary hypertension, vasculitis, collagen vascular disease, chronic rhinosinusitis, immunodeficiency disorders, neutrophil function defects, bronchiolitis, emphysema, bronchiectasis or a hypereosinophilic syndrome. Video stroboscopy did not reveal evidence of laryngeal dysfunction.

Spirometry revealed a postbronchodilator forced expiratory volume in $1 \mathrm{~s}\left(\mathrm{FEV}_{1}\right)$ and forced vital capacity (FVC) of $2.4 \mathrm{~L}(54 \%)$ and

${ }^{1}$ Division of Respirology, Department of Medicine, St Joseph's Healthcare and McMaster University; ${ }^{2}$ Farncombe Family Digestive Health Research Institute, Department of Medicine and Biochemistry and Biomedical Sciences, McMaster University, Hamilton, Ontario

Correspondence: Professor Parameswaran Nair, Firestone Institute for Respiratory Health, St Joseph's Healthcare, 50 Charlton Avenue East,

Hamilton, Ontario L8N 4A6. Telephone 905-522-1155 ext 35044, fax 905-521-6183, e-mail parames@mcmaster.ca 


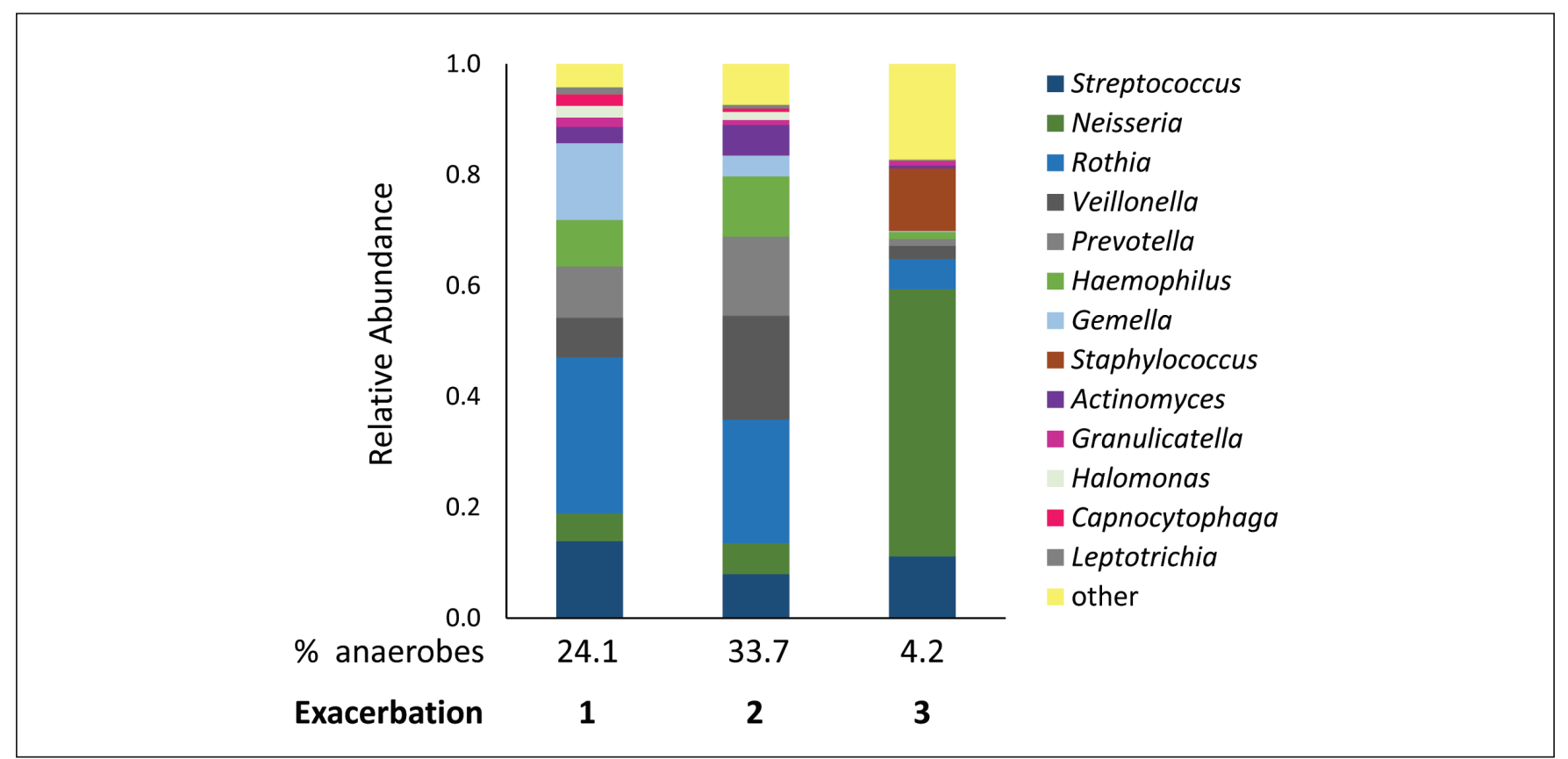

Figure 1) Bacterial composition of sputum from exacerbations. Relative abundance of taxa identified by molecular profiling of the V3 region of the $16 \mathrm{~S}$ ribosomal RNA gene using MiSeq (Illumina, USA) sequencing. Paired end reads were processed through PandaSeq (19) and processed through QIIME (20) as described previously (21). Genera $<1 \%$ were grouped into 'other' to simplify the plot. Obligate anaerobes (Veillonella, Prevotella, Gemella and Leptotrichia) are in grey shades and their total relative abundance is indicated below the plot. Veillonella, Prevotella, Gemella, Rothia and Haemphillus all decrease after exacerbation (2)

$5.3 \mathrm{~L}(97 \%)$, respectively $\left(\mathrm{FEV}_{1} / \mathrm{FVC}\right.$ ratio $\left.46 \%\right)$, which was indicative of moderate airflow obstruction. His previous best $\mathrm{FEV}_{1}$ was $3.2 \mathrm{~L}$ in 2005. The shape of the flow volume curve did not suggest vocal cord dysfunction. He had severe airway hyper-responsiveness with a $\mathrm{PC}_{20}$ methacholine of $0.1 \mathrm{mg} / \mathrm{mL}$. Sputum was induced with hypertonic saline and processed as described previously (3). Quantitative analysis revealed a total cell count of $6.1 \times 10^{6}$ cells/g, with neutrophils accounting for $36 \%$ and eosinophils $0.6 \%$. Exhaled nitric oxide was 12.0 parts per billion. Thus, there was no clinically relevant bronchitis. His medications were left unchanged and he was advised to attend the clinic at his next exacerbation. Accordingly, he was seen urgently one week later for increased cough and wheeze. Similar episodes in the past had been treated with prednisone. At this presentation, his $\mathrm{FEV}_{1}$ had not changed significantly $(2.3 \mathrm{~L})$ while his FVC was 4.7 L. His sputum demonstrated an intensely neutrophilic bronchitis. Total cell count was $129 \times 10^{6}$ cells/g with $97 \%$ neutrophils and $0.3 \%$ eosinophils, which usually indicates bacterial bronchitis. For this exacerbation, he was treated with clarithromycin (Biaxin XL, Abbott, Canada) 500 mg twice daily for two weeks.

The dose of prednisone and inhaled corticosteroids were reduced every six weeks because he did not have an eosinophilic bronchitis. He experienced three exacerbations over the ensuing six months. Sputum at each of these exacerbations showed an intense neutrophilic bronchitis (which was not associated with peripheral blood neutrophilia) but no eosinophils, indicating that the exacerbations were due to infections and unlikely to require treatment with higher doses of corticosteroids. However, standard laboratory cultures did not identify any pathogens. A 23-panel virus polymerase chain reaction study did not identify viruses as causes of his exacerbations. Therefore, extended cultures that included anaerobic and carbon dioxide-enriched conditions were performed and used molecular methods of bacterial detection. Sputum samples were processed as described previously for culture $(4,5)$ and DNA extraction (4). The sputum samples were placed an anaerobic pouch for transfer (GasPak EZ Anaerobe Pouch System, VWR, USA) to the research laboratory. The samples were transferred to an anaerobic chamber and diluted with an equal volume of brain heart infusion medium (BHI) +
$0.05 \%$ L-cysteine. The sample was homogenized by repeated passage through an 18-gauge needle and serial dilutions prepared in BHI + $0.05 \%$ L-cysteine. Samples were plated onto the following media (BHI agar, Columbia Blood agar, Columbia Blood with naladixic acid, Fastidious Anaerobe agar, MacConkey agar, Mannitol Salts agarSA, and Typtic Soy agar with yeast extract (all from Difco, USA) and McKay agar. Replicate plates were incubated under anaerobic conditions and $5 \%$ carbon dioxide at $37^{\circ} \mathrm{C}$ for $48 \mathrm{~h}$ to $72 \mathrm{~h}$. Distinct colony types were quantified and the bacteria identified by sequencing of the 16S RNA gene using standard protocols by partial 16 S ribosomal RNA gene sequencing using of (5'-AGAGTTTGATCCTGGCTCAG-3') and 926r (5'-CCGTCAATTCCTTTRAFTTT-3' by amplification and sequencing of the $\mathrm{V} 3$ region of the $16 \mathrm{~S}$ ribosomal RNA gene using MiSeq (Illumina, USA) $(6,7)$. Taxonomic identification was performed using the Ribosomal Database Project (8), Greengenes (9) and Human Oral Microbiome Database (10). Based on the results, the patient was treated with amoxicillin and clavulanic acid $875 \mathrm{mg}$ twice daily for six weeks, metronidazole $500 \mathrm{mg}$ three times daily for four weeks, $30 \mathrm{~g}$ intravenous immunoglobulin monthly and $5 \%$ saline nebulization daily for 12 months.

Figure 1 shows the microbial community at two exacerbations before and one after therapy. While no obvious pathogens were detected in the first two samples, the sputum was characterized by a polymicrobial colonization including the presence of a significant proportion of obligate anaerobes. The first two samples collected during exacerbations were very similar in composition. The community included a significant burden of obligate anaerobes, most notably Veillonella and Prevotella. The facultative anaerobe Gemella was also a significant member of these communities $(6.6 \%$ and $3 \%$, respectively). These three groups comprised $24.1 \%$ and $33.7 \%$, respectively, of the first two samples but dropped to $4.2 \%$ in the sputum sample after therapy. Also noteworthy was the drop in relative percentage of Rothia, which is an opportunistic pathogen (from 15\% and $17 \%$ in the first to samples to $6 \%$ in the final sample). These results may indicate that improved airway function may correlate with a decrease in growth of anaerobic bacteria and their presence 

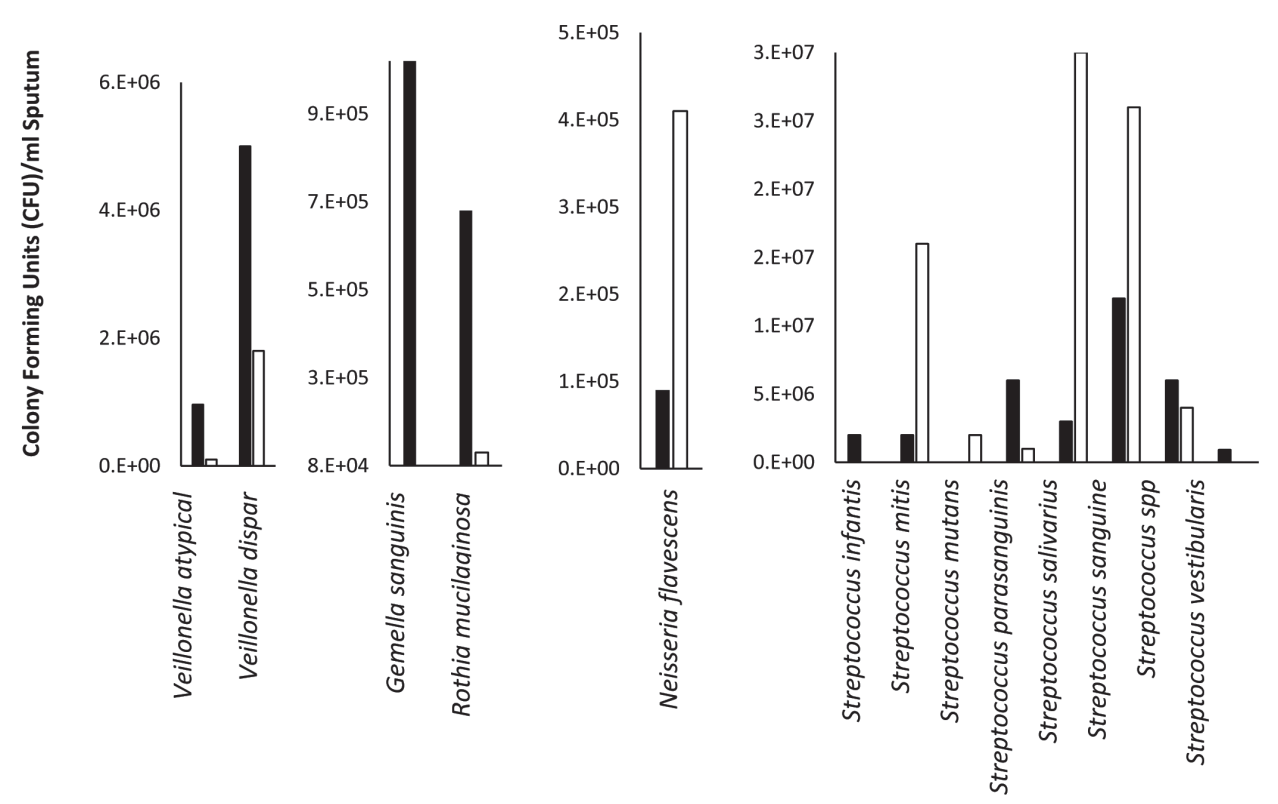

Figure 2) Quantitative culture data for exacerbation samples 2 (solid bars) and 3 (open bars). Decreases in the obligate anaerobes (Veillonella atypical and Veillonella dispar) and facultative anaerobes with pathogenic potential (Gemella sanguinis and Rothia mucilaginosa) and increase in the commensal Neisseria flavescens are observed in the third exacerbation. The heterogeneity in isolated streptococci is also shown. Prevotella was not recovered under the culture conditions used for the samples

may be a biomarker of poor airway health. The third sample showed a marked increase in Neisseria and Staphylococcus. While these genera contain known pathogenic species, the bacteria recovered from these genera by culture were commensal species (Neisseria flavescens, Neisseria mucosa, Neisseria subflava and Staphylococcus epidermidis) (Figure 2).

The effect of this was significant because the number of infective bronchitis episodes decreased from four per year to just one over the following year (indicated as exacerbation 3 in Figure 1). His spirometry remained unchanged $\left(\mathrm{FEV}_{1} 2.35 \mathrm{~L}\right.$ and FVC $\left.4.76 \mathrm{~L}\right)$. Although his general quality of life improved and was able to come off his prednisone and he did not experience exacerbations, he continued to require two to four puffs of salbutamol daily, particularly for exerciseinduced wheeze. In an attempt to further improve his asthma control, bronchial thermoplasty was performed using a procedure that has been previously described (11). He experienced no complications from the procedure. The benefits of the bronchial thermoplasty were noticed objectively and subjectively within one month after the third treatment session. The patient reported feeling the best he ever had, with improved exercise tolerance and his spirometry values increased to an $\mathrm{FEV}_{1}$ of $3.0 \mathrm{~L}(65 \%)$ and an FVC $5.7 \mathrm{~L}(98 \%)$, corresponding to a $\mathrm{FEV}_{1} / \mathrm{FVC}$ ratio of $53 \%$ by the end of the year. His $\mathrm{PC}_{20}$ methacholine has improved to $0.4 \mathrm{mg} / \mathrm{mL}$. He experienced a reduction in the number of infective bronchitis exacerbations, fewer daily symptoms, improved exercise tolerance, decreased yearly clinic visits and a reduced burden of disease, and decreased reliance on medications. He is currently on just $200 \mu \mathrm{g}$ of budesonide/formoterol (Symbicort, AstraZeneca, USA) one puff daily and Oxeze (AstraZeneca, USA) as needed (two to three puffs per week).

\section{DISCUSSION}

The current case presents a unique model for treating patients with severe asthma that is refractory to conventional treatment. The individual aspects of this particular patient's asthma were separated and targeted individually, resulting in a decreased burden of disease and increased quality of life.
This patient was initially being managed with high doses of prednisone for a presumed eosinophilic bronchitis. Through sputum analysis, this was proven to be a neutrophilic inflammatory process. This allowed tailored treatment to wean him off prednisone and alleviate him of the associated side effects, resulting in significant clinical improvement. Identification of the noneosinophilic nature of the exacerbations also avoided him being unnecessarily exposed to the new monoclonal antibody therapies that are currently being developed to target $\mathrm{T}$ helper cell 2-type inflammation. We then attempted to identify the pathogens causing the neutrophilic bronchitis. Routine sputum cultures did not help to direct antibiotic therapy and, therefore, we used molecular microbiological methods (confirmed with extended cultures) that have previously been demonstrated to be useful to treat patients with cystic fibrosis $(4,5)$ and tuberculosis (12). During the patient's exacerbations, the sputum collected showed a high proportion of anaerobic bacteria. Gemella and Rothia species, although not common pathogens, have been implicated in respiratory infections $(13,14)$. This was treated with amoxicillin and metronidazole. The specific benefits of hypertonic saline and intravenous immunoglobulins are not clear but they may have contributed to the altered airway microbiome. Overall there was no significant change in the Shannon diversity index $(2.14,2.43,2.16$ at each time point). A similar rank abundance was also observed, indicating that the general community structure was similar. However, there was a marked decrease in obligate anaerobes and a corresponding increase in commensal Neisseria and Staphylococcus species after treatment with amoxicillin, metronidazole, saline inhalation and intravenous immunoglobulin. Because we could not identify any specific immune deficiency state or neutrophil function abnormality, we speculate that the high doses of corticosteroids may have contributed to the persistent infection with anaerobic bacteria. This is being currently investigated in our laboratory.

Having treated the inflammatory and neutrophilic components of his disease, the patient remained symptomatic, presumably due to severe variable airflow obstruction. Bronchial thermoplasty was thus used to ameliorate his airway hyper-reactivity. Controlled radiofrequency waves 
are applied to the airways through the side channel of a bronchoscope, which reduces airway smooth muscle mass and may lead to reduced bronchoconstriction. The patient reported a significant improvement in his quality of life within one month after the procedure. In patients with severe persistent asthma, thermoplasty has been shown to improve asthma control, as demonstrated by improved peak expiratory flow, scores on the quality of life questionnaires, percentage of symptom-free days and a reduced reliance on rescue medications $(15,16)$, and these benefits are sustained long term (17). It is not currently known whether this improvement in symptoms is, in fact, through attenuation of airway muscle mass (18) and thereby improved airway reactivity.

\section{SUMMARY}

We illustrated the importance of quantitative cell counts in sputum to decrease the dose of steroids when they are not necessary and to suspect infective bronchitis when routine bacterial cultures do not identify

\section{REFERENCES}

1. Canadian Thoracic Society 2012 guideline update: Diagnosis and management of asthma in preschoolers, children and adults: Executive summary. Can Respir J 2012;19e81-8.

2. Hargreave FE, Nair P. Asthma, COPD and bronchitis are just components of airway disease. Eur Respir J 2006;28:264-7.

3. Pizzichini E, Pizzichini MM, Efthimiadis A, Hargreave FE, Dolovich J. Measurement of inflammatory indices in induced sputum: Effects of selection of sputum to minimize salivary contamination. Eur Respir J 1996;9:1174-80.

4. Sibley CD, Parkins M, Duan K, Norgaard CJ, Rabin H, Surette MG. A polymicrobial perspective of pulmonary infections exposes an enigmatic pathogen in cystic fibrosis patients. Proc Natl Acad Sci USA 2008;105:15070-5.

5. Sibley CD, Grinwis ME, Field TR, et al. Culture enriched molecular profiling of the cystic fibrosis airway microbiome. PLoS One 2011;6:e22702

6. Bartram AK, Lynch MD, Stearns JC, Moreno-Hagelsieb G, Neufeld JD. Generation of multimillion-sequence 16S rRNA gene libraries from complex microbial communities by assembling paired-end illumina reads. Appl Environ Microbiol 2011;77:3846-52.

7. Stearns JC, Lynch MD, Senadheera DB, et al. Bacterial biogeography of the human digestive tract. Sci Rep 2011;1:170.

8. Cole JR, Wang Q, Cardenas E, et al. The Ribosomal Database Project: Improved alignments and new tools for rRNA analysis. Nucl Acids Res 2009;37(Suppl 1):D141-D145.

9. DeSantis TZ, Hugenholtz P, Larsen N, et al. Greengenes, a Chimera-Checked 16S rRNA Gene Database and Workbench Compatible with ARB. Appl Environ Microbiol 2006;72:5069-72.

10. Chen T, Yu W-Han, Izard J, Baranova OV, Lakshmanan A, Dewhirst FE. The Human Oral Microbiome Database: A web accessible resource for investigating oral microbe taxonomic and genomic information. Database 2010; Vol 2010, Article ID baq013, doi: 10.1093/database/baq013.

11. Cox G, Thomson NC, Rubin AS, et al. Asthma control during the year after bronchial thermoplasty. N Engl J Med 2007;356:1327-37. pathogens. The present case history also demonstrates the evolving utility of applying molecular methods to detect airway pathogens and the significant impact of bronchial thermoplasty on the quality of life of patients with severe asthma when the procedure was performed after adequate control of airway inflammation. The present report also highlights the importance of characterizing the pathophysiological nature of individual exacerbations rather than considering all of them as uniform.

DISCLOSURES: Dr Nair is supported by a Canada Research Chair in Airway Inflammometry. Dr Nair is listed on a patent for a sputum filtration device and has provided scientific advice to Cellometrics, a University spin-off company. GC has received funding and honorarium for consultation from Boston Scientific. CC, MK and MGS do not have any financial disclosures of conflicts of interest related to this article.

12. Chowdhury IH, Aditi S, Bojlul B, et al. A molecular approach to identification and profiling of first-line-drug-resistant mycobacteria from sputum of pulmonary tuberculosis patients. J Clini Microbiol 2012;50:2082-4.

13. Sibley CD, Parkins M, Field TR, et al. The genus Gemella: Commensals or pathogens in cystic fibrosis? North American Cystic Fibrosis Conference. Pediatr Pulmonol 2008;(Suppl)31:356.

14. Hiyamuta H, Tsuruta N, Matsuyama T, Satake M, Ohkusu K, Higuchi K. First case report of respiratory infection with Rothia aeria. (Article in Japanese) Nihon Kokyuki Gakkai Zasshi 2010;48219-23.

15. Pavord ID, Cox G, Thomson NC, et al. Safety and efficacy of bronchial thermoplasty in symptomatic, severe asthma. Am J Respir Crit Care Med 2007;176:1185-91.

16. Castro M, Rubin AS, Laviolette M, et al. Effectiveness and safety of bronchial thermoplasty in the treatment of severe asthma: A multicenter, randomized, double-blind, sham-controlled clinical trial. Am J Respir Crit Care Med 2010;181:116-24.

17. Wechsler ME, Laviolette M, Rubin AS, et al. Bronchial thermoplasty: Long-term safety and effectiveness in patients with severe persistent asthma. J Allergy Clin Immunol 2013 Aug 30. doi:pii: S0091-6749(13)01268-2. 10.1016/j.jaci.2013.08.009.

18. Pretolani M, Dombret MC, Thabut G, et al. Reduction of airway smooth muscle mass by bronchial thermoplasty in patients with severe asthma. Am J Respir Crit Care Med 2014;190:1452-4.

19. Masella AP, Bartram AK, Truszkowski JM, Brown DG, Neufeld JD. PANDAseq: Paired-end assembler for illumina sequences. BMC Bioinformatics 2012;13:31.

20. Caporaso JG, Kuczynski, J Stombaugh J, et al. QIIME allows analysis of high-throughput community sequencing data. Nat Methods 2010; doi: 10.1038/nmeth.f.303

21. Whelan FJ, Verschoor CP, Stearns J, et al. The loss of topography in the microbial communities of the upper respiratory tract in the elderly. Ann Am Thorac Soc 2014;11:513-21. 


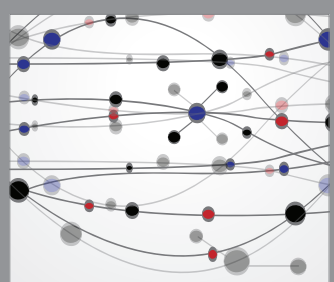

The Scientific World Journal
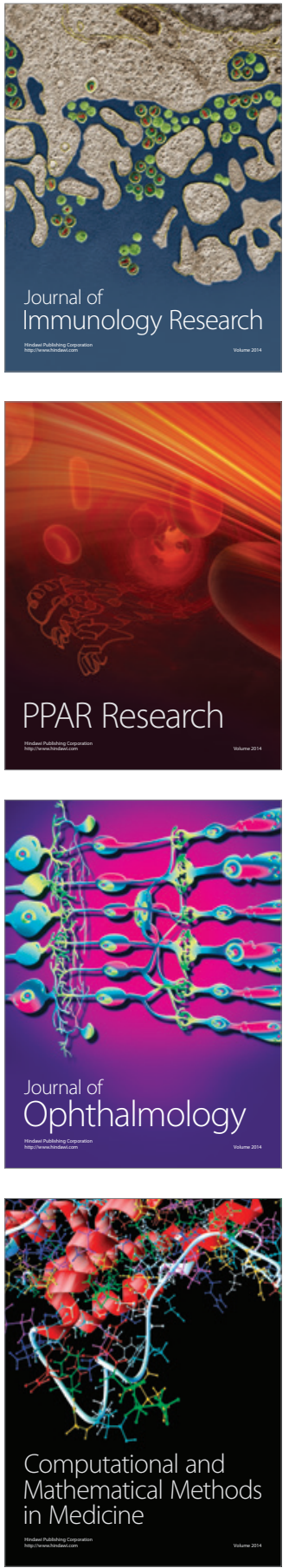

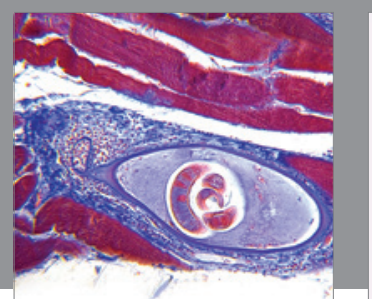

Gastroenterology Research and Practice

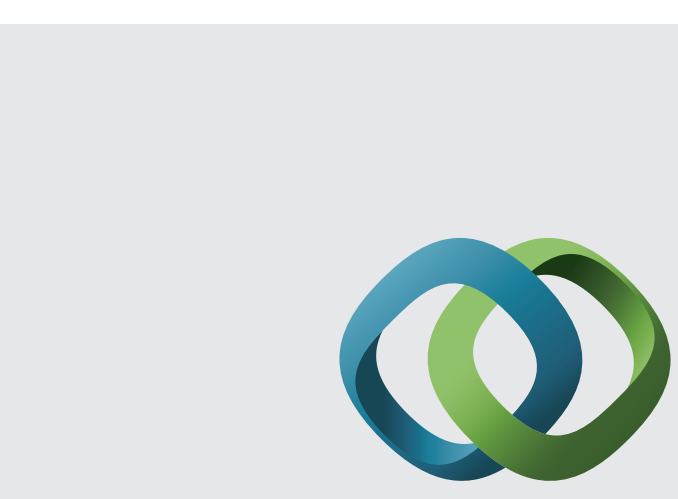

\section{Hindawi}

Submit your manuscripts at

http://www.hindawi.com
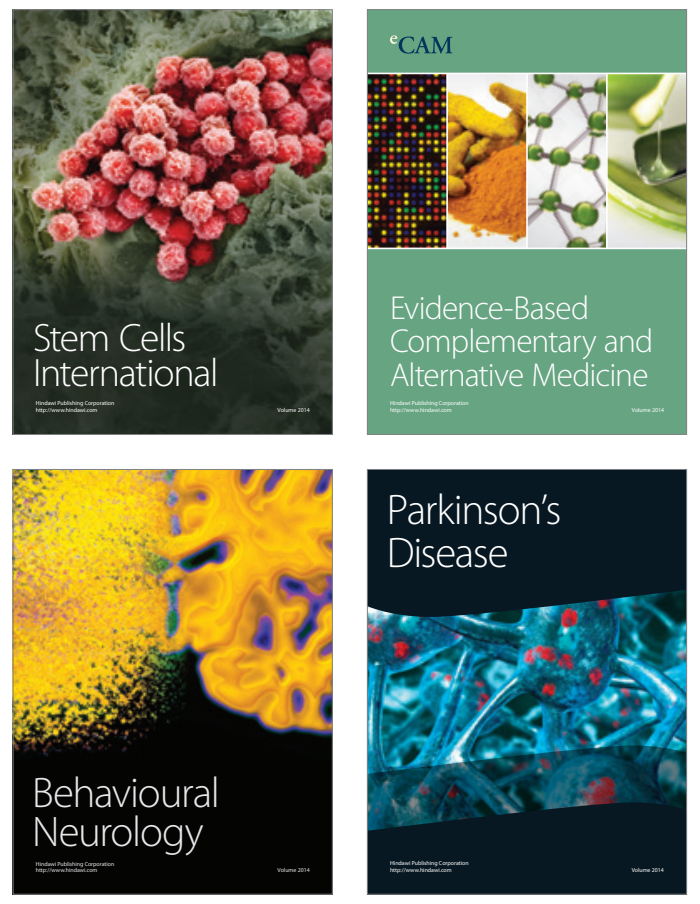
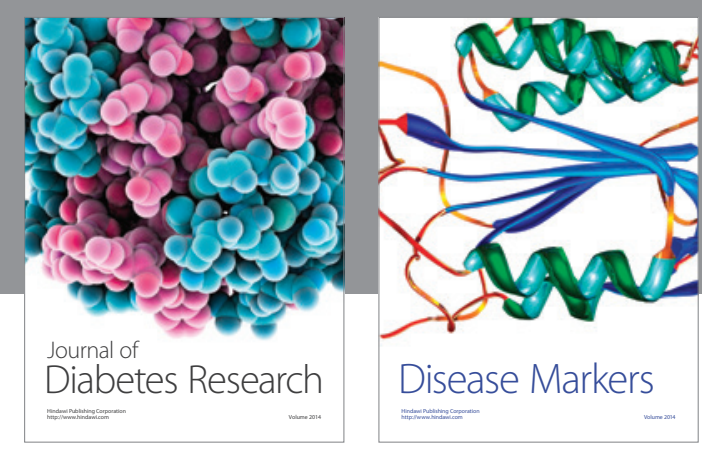

Disease Markers
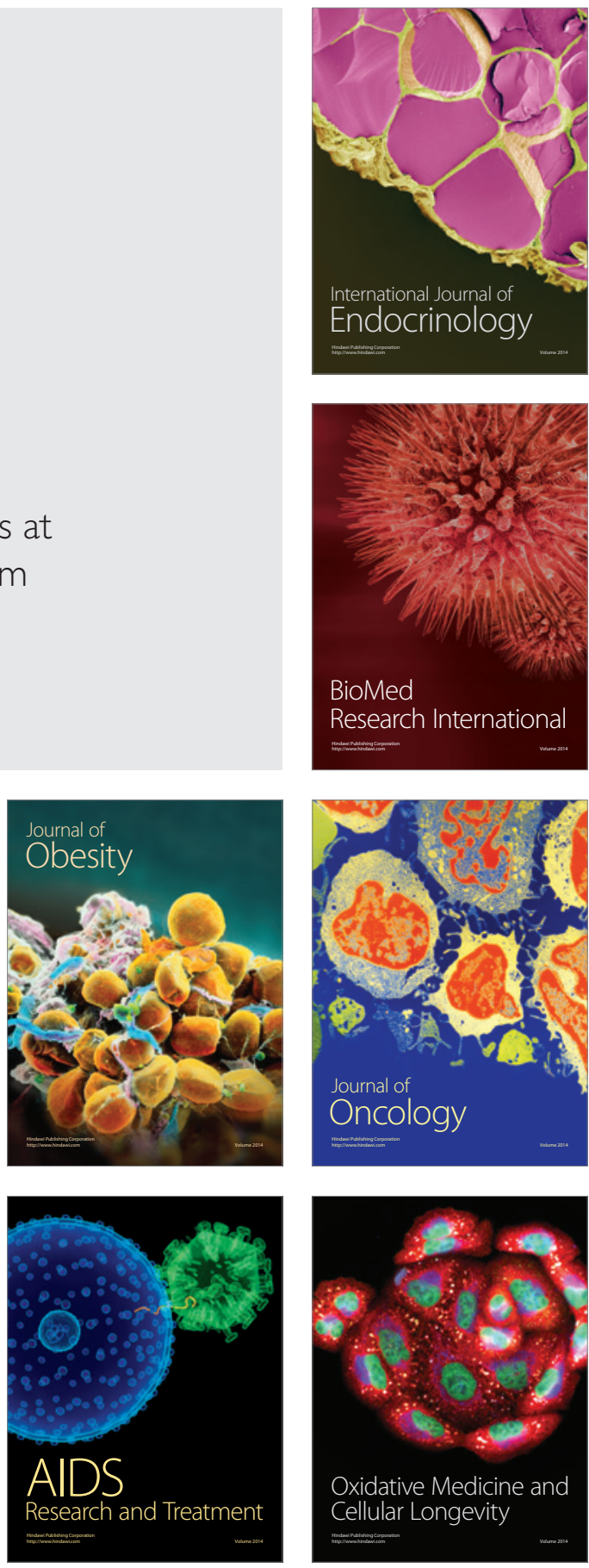